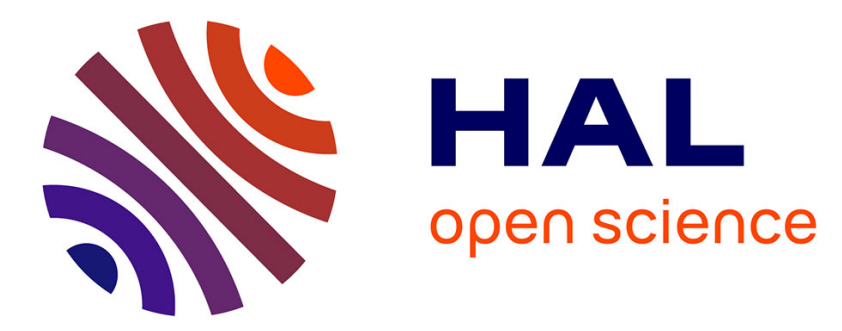

\title{
Les éthiques du partage des avantages dans la gouvernance internationale de la biodiversité sauvage et cultivée
}

Frédéric Thomas

\section{- To cite this version:}

Frédéric Thomas. Les éthiques du partage des avantages dans la gouvernance internationale de la biodiversité sauvage et cultivée. Revue d'éthique publique, 2014, Volume 16 (Numéro 1), pp.181-198. ird-01018838

\section{HAL Id: ird-01018838 \\ https://hal.ird.fr/ird-01018838}

Submitted on 6 Jul 2014

HAL is a multi-disciplinary open access archive for the deposit and dissemination of scientific research documents, whether they are published or not. The documents may come from teaching and research institutions in France or abroad, or from public or private research centers.
L'archive ouverte pluridisciplinaire HAL, est destinée au dépôt et à la diffusion de documents scientifiques de niveau recherche, publiés ou non, émanant des établissements d'enseignement et de recherche français ou étrangers, des laboratoires publics ou privés. 


\title{
LES ÉTHIQUES DU PARTAGE DES AVANTAGES DANS LA GOUVERNANCE INTERNATIONALE DE LA BIODIVERSITÉ SAUVAGE ET CULTIVÉE
}

\author{
FRÉDÉRIC THOMAS ${ }^{1}$ \\ Institut de recherche pour le développement
}

Résumé: Les mécanismes d'accès aux ressources génétiques et de partage des bénéfices qui découlent de leur mise en valeur reposent en droit international sur des conceptions de la justice très différentes selon que l'on se place dans le cadre de la Convention sur la diversité biologique (CDB) ou dans celui du traité international de la FAO sur les ressources génétiques agricoles (Tirpaa). Le présent article montre que l'éthique marchande contractuelle de la CDB relève plutôt d'une conception rawlsienne de la justice en admettant que des inégalités peuvent être justes si elles donnent lieu à des compensations, tandis que la volonté de la FAO de faire des ressources génétiques un bien public mondial pour en faciliter l'accès dans un système mutualisé s'apparente à une conception libertarienne de la justice privilégiant la liberté d'opérer des industriels et des chercheurs.

Face aux limites de la biosphère, qui remettent en cause nos modèles de développement économique et qui nous engagent dans de nouveaux principes de responsabilité (Jonas, 1979), la distribution équitable des avantages que les hommes tirent de la nature et des désavantages que

1. Je remercie chaleureusement Catherine Larrère pour la relecture d'une première version du présent article, ainsi que les relecteurs de la revue Éthique publique pour leurs critiques et propositions. 
génère son exploitation est devenue un enjeu d'éthique globale liant éthiques de l'environnement et justice environnementale ${ }^{2}$. Le présent article se limite à un point particulier de cette très vaste question. Il étudie les différentes conceptions de la justice qui sous-tendent un mécanisme central à la gouvernance internationale de la biodiversité : les règles d'accès aux ressources génétiques et le partage des avantages qui découlent de leur mise en valeur. Les deux grands régimes juridiques qui organisent à l'échelle internationale cet accès et ce partage seront examinés. Le premier est produit par la Convention sur la diversité biologique (CDB). Celle-ci énonce que le pourvoyeur d'une ressource génétique doit pouvoir profiter des bénéfices qui résultent de sa mise en valeur par autrui. Le deuxième est construit par le Traité international de la FAO sur les ressources phytogénétiques pour l'agriculture et l'alimentation (Tirpaa). Celui-ci institue, d'un côté, un régime de mutualisation de ces ressources entre les États membres, particulièrement pour la recherche en amélioration des plantes, et affirme, de l'autre, que les agriculteurs ont des droits sur les plantes qu'ils cultivent au nom de leur contribution passée, présente et future à l'entretien de la biodiversité cultivée.

De nombreux auteurs ont déjà montré que différentes conceptions de la justice issues des théories en sciences politiques étaient au cœur des grandes conventions environnementales internationales (Dobson, 1998 et

2. On entend par éthiques de l'environnement toutes les réflexions qui visent à étendre les principes moraux de respect de la personne aux non-humains : les animaux, les végétaux, mais aussi les communautés biotiques, les écosystèmes ou la géosphère tout entière, en attribuant à ces entités différentes formes de valeurs; on oppose généralement la valeur intrinsèque aux valeurs instrumentales selon qu'on se situe dans des éthiques anthropo-, éco- ou bio-centrées de la nature ou encore pragmatistes (Larrère, 1997; Callicott, 2010; Hache, 2011; Hess, 2013 ; etc.). La justice environnementale se consacre de son côté à l'étude de la juxtaposition des inégalités socioéconomiques et écologiques et aux conceptions de la justice à mobiliser pour réintroduire de l'équité dans la répartition des inégalités environnementales (Okereke, 2006; Blanchon et al., 2009). Bien que présente en filigrane tout au long de notre exposé, la question très discutée de la compatibilité entre éthique de l'environnement et justice environnementale ne sera pas l'objet central du présent article. Signalons simplement que de nombreux travaux ont souligné l'ignorance mutuelle des deux champs, mais aussi la possibilité de les rendre «mutuellement fonctionnels " (voir Dobson, 1998). Catherine Larrère suggère de son côté que, quelle que soit la conception de la justice adoptée, on ne puisse «parler de justice environnementale qu'en renonçant à l'éthique environnementale, du moins à celle qui fait de la valeur intrinsèque son argument central» (2009: 162). Adrian Armstrong considère du sien que, en dépit de l'anthropocentrisme de la justice environnementale et des formes de militantisme qui s'y rattachent, il existe plusieurs "points de contact» entre éthique et justice environnementale, particulièrement dans le contexte d'actions conjointes de lutte contre les inégalités environnementales et les atteintes à l'environnement (Armstrong, 2014). 
1999; Benton, 1997 et 1999; Langhelle, 2000; Okereke, 2006). Cinq conceptions de la justice sont particulièrement retenues dans ces travaux:

1. La conception utilitariste de la justice qui consiste à chercher la maximisation du bonheur du plus grand nombre possible de personnes;

2. La conception libérale égalitaire qui admet les inégalités sociales et économiques si elles reposent sur une égalité de droits qui fonde l'égalité des chances. Dans ces conditions, il peut exister des inégalités justes, c'est-à-dire bénéfiques aux éléments les plus fragiles la société (Rawls, 1971)

3. La conception communautariste qui considère qu'il ne peut $y$ avoir de justice sans prise en compte de la spécificité des valeurs culturelles de la justice (Sandel, 1982; Young, 1990);

4. La conception égalitarienne de la satisfaction des besoins de chacun qui fait de la justice un impératif catégorique kantien qui consiste à «traiter les personnes comme des fins en soi dignes », et pas seulement comme les moyens de réaliser une justice supérieure (Viotti et Kauppi, 1987: 520);

5. La conception libertarienne qui considère que ce qui est juste, c'est que les gens aient avant tout droit à leurs parts de marché, quel que soit le degré de besoin ou la pauvreté qui les entoure (Nozick, 1974).

La plupart des auteurs s'accordent à dire que la conception utilitariste n'est guère compatible avec des objectifs environnementaux. Beaucoup soutiennent, en revanche, que la conception égalitarienne est au centre du concept de développement durable puisque, en conditionnant la durabilité à la lutte contre la pauvreté, le rapport Brundtland fait de la satisfaction des besoins de chacun un instrument central de la conservation de la nature. C'est un exemple de «relations fonctionnelles» entre éthique et justice environnementale telles que pensées par Dobson (1999).

3. Pour que la somme des inégalités sociales soit bénéfique aux plus pauvres, la théorie de John Rawls suppose qu'en l'absence d'un certain degré d'inégalité qui produit des riches, les pauvres seraient encore plus pauvres en profitant moins de la richesse des riches. 
Néanmoins, il semble que ce soit la conception libérale égalitaire qui soit la plus prégnante dans le droit international de l'environnement. Elle est à la base de tous les principes de compensation qui admettent que des inégalités environnementales peuvent être justes si les richesses qu'elles produisent génèrent des compensations. C'est à partir de cette conception de la justice que la convention climat, par exemple, impose une distribution des ressources selon laquelle les nations qui ont utilisé plus que leur part des ressources naturelles globales doivent compenser celles qui en ont moins utilisé. Il y a ainsi une reconnaissance internationale qu'il existe une certaine dette environnementale du Nord envers le Sud qui mérite compensation. La convention de Bâle sur la circulation internationale et le traitement des déchets est quant à elle plutôt fondée sur une conception libertarienne de la justice. En effet, même si elle a sans doute permis de limiter l'attitude des pays riches de déverser leurs déchets dangereux dans des pays pauvres qui nont pas la capacité de les traiter (Puckett, 1992: 9394), elle assume la violence de l'idée qu'interdire ces occasions d'affaires serait injuste envers les pays qui en ont besoin pour leur développement quand bien même ces marchés se construisent au prix d'une morbidité décuplée d'une partie de leur population généralement non informée des conséquences sanitaires pour elle-même (Okereke, 2006 : 732).

Par ces quelques exemples, on comprend donc que le droit de l'environnement peut être fondé sur une diversité de conceptions de la justice. Dans les développements qui suivent, nous allons précisément mettre en lumière les différentes conceptions de la justice que nous pensons à l'œuvre dans les deux régimes d'accès à la biodiversité et de partage des avantages qui en découlent que nous avons évoqués.

\section{LA CONCEPTION DU PARTAGE DANS LE RÉGIME D'APPROPRIATION DE LA BIODIVERSITÉ DE LA CBD}

\section{LA CONCEPTION RAWLSIENNE DE LA JUSTICE DE LA CDB}

La Convention sur la diversité biologique (CDB) fait de l'accès aux ressources génétiques et aux savoirs traditionnels des communautés autochtones et locales et du partage des avantages qui découlent de la mise en valeur de ces ressources ou de ces connaissances, l'un de ses trois objectifs à égalité avec la conservation de la biodiversité et l'utilisation durable de 
ses éléments (article 1). Le partage des avantages vise à rétablir une certaine équité entre les laboratoires et les industries qui réalisent de substantiels bénéfices par la mise en valeur de ressources naturelles et les populations qui fournissent ces ressources sans tirer de bénéfices des innovations qui en découlent. La conception de la justice qui préside à cet édifice est essentiellement rawlsienne. L'idée est que par des contrats d'accès aux ressources génétiques librement consentis entre deux parties également informées, on peut atteindre la meilleure distribution des avantages que l'humanité peut tirer de la biodiversité et par voie de conséquence la meilleure conservation de la ressource. La redistribution des bénéfices auprès des populations locales est conçue pour que celles-ci soient en effet mieux en mesure de conserver leur environnement. L'éthique rawlsienne permet clairement, ici, de réaliser conjointement une certaine justice environnementale et une certaine éthique de l'environnement (du moins en théorie).

Si l'on rentre dans la technicité des outils que la CDB propose pour mettre en œuvre ces objectifs croisés, on peut dire que la CDB tente d'instituer à l'échelle internationale un régime contractuel d'accès et de partage des avantages (APA) entre fournisseurs et utilisateurs de ressources génétiques. L'article 15 précise que l'accès aux ressources génétiques doit être soumis au «consentement préalable informé » (CPI) de l'État pourvoyeur de la ressource et doit se faire selon des «termes mutuellement convenus » (TMC). Il réclame des Parties (c'est-à-dire les États membres de la CDB) de mettre en place "des mécanismes dans les législations nationales qui permettent le partage des avantages de l'utilisation d'une ressource génétique avec l'État qui a fourni ladite ressource» (article 15.7). Les dispositions de la $\mathrm{CDB}$ en matière d'accès aux connaissances traditionnelles sont moins contraignantes. L'article $8 \mathrm{j}$ encourage le respect, la préservation et la diffusion des savoirs traditionnels ainsi que le partage des avantages découlant de l'utilisation de ces savoirs avec les communautés autochtones et locales qui en sont détentrices. Le protocole de Nagoya (signé au Japon le 30 octobre 2010, à l'occasion de la $10^{\mathrm{e}}$ Conférence des Parties de la CDB) est venu renforcer ces mécanismes. Il oblige notamment les Parties à s'assurer que les ressources génétiques exploitées sur leur territoire ont été acquises en conformité avec la législation du pays fournisseur. Notamment, les Parties vérifient que leurs ressortissants disposent du ou des CPI, examinent les TMC et les accords de partage conformes à la législation du pays 
fournisseur (article 15), de même en ce qui concerne l'accès aux savoirs traditionnels (article 16). Les Parties doivent pour cela mettre en place un ou des points de contrôle (PC) (article 17.1) qui sont chargés de collecter toute l'information pertinente "à tous les stades de la recherche, du développement, de l'innovation, de la précommercialisation ou de la commercialisation»(article 17.1 a) iv)). C'est un renforcement important de la conception rawlsienne de la justice initiale de la $\mathrm{CDB}$, dans la mesure où le protocole de Nagoya vient ajouter de nombreux instruments institutionnels que les pouvoirs publics nationaux vont devoir mettre en place pour que le partage des avantages constitue un vrai instrument d'équité.

Il faut souligner combien ce cadre contractuel d'APA a constitué un tournant important dans l'économie des ressources génétiques. Jusque-là, la biodiversité était considérée à l'échelle internationale comme un patrimoine commun de l'humanité, un bien public mondial, dans lequel les industries et la recherche puisaient en général sans contrepartie (Thomas, 2006), multipliant ainsi ce que les pays en développement et les organisations non gouvernementales internationales ont dénoncé comme des actes de biopiraterie (Shiva, 1997). Cette pratique du libre accès aux ressources génétiques se faisait au nom de l'intérêt général: le progrès scientifique et technique que cet accès libre et gratuit était censé apporter à l'ensemble de l'humanité. Mais du point de vue des conceptions libérales de la justice, ce régime du libre accès consistait finalement à confier la réalisation de la justice à la liberté de chacun d'avoir sa part de ressources génétiques, ce qui s'apparentait fortement à une conception libertarienne de la justice. L'accès contractualisé institué par la CDB a donc permis de sortir de ce libertarisme pour y substituer une conception plus rawlsienne.

Cette conception de la justice à l'avantage des pays riches en biodiversité n'a pas été sans contrepartie pour les pays riches en technologies. En effet, le principe de partage des avantages de la CDB a joué un rôle fondamental pour que les pays du Sud reconnaissent la brevetabilité du vivant, car réclamer le partage des bénéfices supposait implicitement reconnaître les outils de propriété industrielle permettant de réaliser des profits sur les innovations qui découlent de l'accès aux ressources. C'est l'aboutissement logique de la conception rawlsienne de la justice qui considère qu'une injustice (les brevets détenus à $95 \%$ par les grandes firmes des pays riches) est acceptable si elle permet une redistribution 
auprès des plus défavorisés. La CDB fait d'ailleurs des brevets l'un des avantages qui découlent de l'accès aux ressources génétiques, et plus encore, un avantage qui doit être respecté par les Parties et qui constitue une source essentielle du partage des avantages, notamment sous la forme de transferts facilités de la biotechnologie brevetée 4 . La CDB soutient ainsi que les biotechnologies agricoles brevetées, donc les organismes génétiquement modifiés, contribuent à la conservation de la biodiversité, à l'utilisation durable de ses éléments et au partage des avantages avec les populations.

Signalons pour finir que ce schéma général n’a pas donné lieu en vingt ans à la multiplication espérée de contrats de bioprospection entre les pays en développement et les laboratoires des firmes pharmaceutiques et des biotechnologies agricoles permettant au principe de partage des avantages de devenir la clef de voûte de l'éthique du «biocapitalisme» naissant. Seuls 39 pays sur les 193 Parties à la CDB ont établi des législations nationales sur l'accès et le partage des avantages, essentiellement des pays en développement (Buck et Hamilton, 2011: 48). Les contrats de bioprospection sont peu nombreux et rarement le résultat de négociations directes entre une firme biotechnologique et des communautés locales dans l'objectif de mettre en valeur la biodiversité et les savoirs traditionnels associés. Ils sont surtout passés entre organismes publics ou dans le cadre de consortiums public-privé qui se déclarent à but non commercial et concernent majoritairement des pays d'Amérique latine (Vivas-Eugui, 2012: 10). Les explications sont nombreuses. Les économistes ont notamment bien montré que la biodiversité entrait mal dans la théorie du marché (Boisvert et Caron, 2007) et les anthropologues ont fait le constat que le partage des avantages construisait artificiellement les contours des communautés récipiendaires des retours, sans englober la totalité des collectifs assurant la conservation de la biodiversité et sans prendre en compte la totalité des valeurs d'usage et de non-usage de la biodiversité pour les populations locales (Hayden, 2003 et 2007) ${ }^{5}$.

4. L'article 1 affirme en effet que le transfert approprié de techniques pertinentes constitue un moyen privilégié pour assurer non seulement le partage des avantages, mais aussi la conservation de la biodiversité et l'utilisation durable des ressources génétiques; puis l'article 16 affirme que le terme technologie inclut la biotechnologie et que les brevets les protégeant doivent être respectés.

5. Cori Hayden oppose au paradigme de l'individu moral comme unique sujet possible du contrat de partage des bénéfices, lidée qu'en matière de bioéthique les consentements préalables informés 


\section{LA CONCEPTION DU PARTAGE DANS LE RÉGIME DE MUTUALISATION DES RESSOURCES GÉNÉTIQUES DE LA FAO}

À rebours du régime marchand contractuel de la $\mathrm{CDB}$, la $\mathrm{FAO}$ considère que les ressources phytogénétiques pour l'agriculture et l'alimentation (ciaprès ressources génétiques agricoles) sont des ressources particulières dont dépend la sécurité alimentaire mondiale et qu'elles ne peuvent donc pas être gouvernées à l'échelle internationale dans un cadre contractuel comme le reste de la biodiversité. Depuis 1992, en réponse au régime d'accès et de partage de la $\mathrm{CDB}$, la $\mathrm{FAO}$ a donc travaillé à refonder les ressources génétiques agricoles en une sorte de «commun scientifique» dont l'accès est ouvert (gratuit) sur la base de l'échange mutuel, comme cela était l'usage avant la signature de la $\mathrm{CDB}$, quand les ressources génétiques agricoles étaient proclamées être «un patrimoine commun de l'humanité» (Engagement international de la FAO, 1983) (Fowler et Hodgkin, 2004 ; Thomas, 2006).

\section{LES INSTRUMENTS DU TIRPAA}

L'aboutissement de ce travail est en 2001 l'adoption du Traité international pour les ressources phytogénétiques pour l'agriculture et l'alimentation (Tirpaa). L'article $1 \mathrm{du}$ Tirpaa, qui prend acte du régime contractuel de la $\mathrm{CDB}$ et affirme travailler en conformité avec son principe de partage des avantages, proclame la nécessité de créer un régime sui generis d'accès aux ressources génétiques pour l'agriculture et l'alimentation des 64 espèces agricoles de son annexe 1 . Le Tirpaa met ainsi en place un système multilatéral qui vise à faciliter la circulation des ressources agricoles entre les pays membres du traité et en somme à lever les contraintes et difficultés d'un accès contractualisé. Tout État qui verse des ressources génétiques dans ce système multilatéral a ainsi accès à toutes les ressources que les autres pays y ont mis; il s'engage en retour à laisser les ressources génétiques acquises grâce au système multilatéral en libre accès pour tout

(re)construisent des collectifs comme sujet éthique souverain, mais dont les contours sont extrêmement problématiques à arrêter, la question du partage des avantages n'étant pas une question de don et de récompense, mais un problème «d'agencements distributifs» (Hayden, 2007: 744 sqq.). 
autre utilisateur (article 12.3.3) ${ }^{6}$. Ce faisant, le Tirpaa répond aux pratiques des obtenteurs de variétés nouvelles qui ont besoin de laisser les ressources génétiques en libre accès pour multiplier les croisements par différentes techniques. Ceux-ci ne revendiquent pas de droits de propriété intellectuelle sur les ressources génétiques, mais sur les résultats de ces croisements au niveau de la plante entière fixée en une combinaison particulière d'allèles, c'est-à-dire sur la variété végétale DHS (distincte, homogène, stable), objet juridique protégeable par un droit de propriété intellectuelle différent du brevet : le certificat d'obtention végétale (COV) mis en place en 1961 par la Convention UPOV (l'Union internationale pour la protection des obtentions végétales) (Thomas, 2006 ; Bonneuil et Thomas, 2009). On a donc deux régimes d'accès qui sont très différents selon qu'on se place dans le cadre de la CDB ou du Tirpaa, particulièrement du point de vue de la mise en œuvre du principe de partage, reconnu par le Tirpaa mais pensé d'une tout autre manière que dans la CDB.

Le Tirpaa prévoit que les modalités de partage des avantages sont précisées au moment de l'accès facilité au système multilatéral dans l'accord de transfert de matériel (ATM). Chaque échange de matériel génétique est en effet le sujet d'un ATM qui peut être assorti d'un accord de partage entre les organismes fournisseurs et récipiendaires. Par cette disposition, la FAO veille à être en conformité avec la reconnaissance du principe de partage des avantages désormais admis internationalement. Mais en proposant de substituer aux contrats de partage (et leur cohorte d'outils institutionnels : CPI, TMC, APA...) un document administratif standardisé de transfert matériel, la FAO sort du cadre contractuel de la $\mathrm{CDB}$ et de la conception rawlsienne qui y préside. La question se pose donc de savoir sur quelle conception de la justice repose la circulation des ressources génétiques dans le Tirpaa.

6. Cette règle de ne pas déposer de droit de propriété intellectuelle clôturant l'accès à la ressource génétique acquise dans le cadre du système multilatéral n'est pas nouvelle. On la trouve clairement énoncée par le Consultative groupe de recherche agricole internationale (CGIAR) dès les années 1990 (Anderson, 2008: 93). 


\section{À QUI PROFITE LA MUTUALISATION DES RESSOURCES ?}

Dans l'esprit du Tirpaa, le partage des avantages, c'est avant tout le système multilatéral lui-même. L'article 13 dit explicitement que ce qu'il faut entendre par avantage est «l'accès facilité » lui-même, c'est-à-dire l'accès gratuit aux ressources génétiques versées dans le système multilatéral. Il n’y a donc plus de rapport contractuel proprement dit pour partager des avantages négociés, mais un avantage général à jouer une mise en commun multilatéral. Du point de vue de l'équité du régime ainsi mis en place, la question nest plus alors de savoir «qui partage quoi avec qui ( (les bénéfices tirés de la brevetabilité du vivant entre les industriels et les pourvoyeurs de la ressource), mais de comprendre à qui profite la mutualisation des ressources. Or, les principaux utilisateurs du système multilatéral ne sont pas les paysans qui ont créé et conservé la biodiversité agricole de génération en génération, mais les scientifiques et les industriels pour qui ce "pot commun» est conçu. Selon cette conception, on peut bien sûr considérer que les agriculteurs sont inclus dans le partage des avantages en tant qu'utilisateurs finaux des variétés industrielles. Mais un tel raisonnement consiste à dire aux communautés rurales que le partage des avantages, c'est le progrès scientifique général. Ce raisonnement constitue un retour vers une conception impérialiste de la justice par rapport à ce que le Sommet de Rio avait réussi à imposer, sans compter que ce "progrès scientifique", autrefois pensé comme un bien public sous la forme des variétés améliorées de la révolution verte, est désormais payant sous la forme de variétés privées de plus en plus chères.

Cette conception est de surcroît en forte contradiction avec la place centrale que le Tirpaa accorde, par ailleurs, aux agriculteurs dans la conservation de la biodiversité cultivée. Le préambule du Tirpaa reconnaît en effet que les agriculteurs ont des droits sur les ressources génétiques agricoles au nom de leurs « contributions passées, présentes et futures [...] à la conservation, l'amélioration et la mise à disposition de ces ressources " et il admet que la «concrétisation de ces droits des agriculteurs» réside fondamentalement dans «les droits [...] de conserver, utiliser, échanger et vendre des semences de ferme et d'autres matériels de multiplication ». En somme, la $\mathrm{FAO}$ suggère que les droits des agriculteurs à produire librement leurs semences à partir des variétés protégées par un COV ou un brevet devraient pouvoir constituer un mécanisme du partage juste et 
équitable des avantages au titre de leur participation passée, présente et future à la conservation de la biodiversité agricole dans laquelle les sélectionneurs puisent pour mettre au point les variétés industrielles à haut rendement. Malheureusement, l'exercice de ce droit est désormais limité au périmètre de l'exploitation agricole dans la plupart des pays sous l'influence des lobbies semenciers qui travaillent au renforcement de leurs droits de propriété industrielle sur les variétés et les semences ${ }^{7}$. Or, rien dans la suite du texte du Tirpaa ne vient contraindre les États à mettre en place des dispositions limitant cette tendance à l'exclusivisme de la propriété industrielle sur les variétés protégées ${ }^{8}$.

Le Tirpaa prévoit cependant pour les agriculteurs la mise en place d'un fonds fiduciaire de partage des avantages (FPA) pour financer des programmes de conservation in situ avec les paysanneries pauvres des pays en développement. Ce fonds de partage est alimenté pour partie par une taxation des produits commercialisés à partir de ressources génétiques issues du système multilatéral. Tout utilisateur d'une ressource acquise grâce à ce système qui en tire une innovation protégée par un droit de propriété intellectuelle doit verser à ce fonds de partage « une part équitable des avantages découlant de la commercialisation de ce produit» (article 13). Cependant, si la ressource en question reste « disponible sans restriction pour d'autres bénéficiaires à des fins de recherche et de sélection ", ce qui est le cas des variétés protégées par un COV, l'utilisateur n'est contraint à aucun versement (suite de l'article 13). Les détenteurs de $\mathrm{COV}$, qui sont les principaux utilisateurs du système multilatéral, ne sont donc pas contraints de verser au fonds de partage du Tirpaa. Ce sont par conséquent les États qui assurent l'essentiel du financement du FPA. Une vingtaine de projets d'un montant maximal de 50000 dollars américains ont ainsi pu être financés depuis 2009. Si exemplaire que soit chaque programme pris individuellement (comme le Parc de la pomme de terre au Pérou), on mesure ici la modestie du mécanisme par rapport aux milliards

7. Voir l'article 15.2 de la Convention UPOV de 1991; pour la France, voir la loi du 8 décembre 2011 qui limite fortement le droit des agriculteurs de produire leurs semences.

8. Sur l'exclusivisme du droit des brevets dans le domaine végétal, voir Hermitte et Kahn (2004: 57 -

63) et Girard et Noiville (2013); sur les tendances récentes à l'exclusivisme du droit UPOV sous les effets du développement des brevets dans le secteur de l'amélioration des plantes, voir Thomas et Boisvert (à paraître, chapitre 3). 
de dollars que la circulation des ressources génétiques permet d'engendrer tant pour les industries semencières conventionnelles que pour les multinationales des biotechnologies.

La FAO, qui essaie de faire du FPA du Tirpaa et de ces quelques projets de conservation in situ de l'agrobiodiversité la vitrine de l'équité du système multilatéral envers les agriculteurs, masque en fait un retour au grand principe du libre accès donnant aux entreprises semencières toute la liberté d'opérer qu'elles réclament. Ainsi, le Tirpaa, qui prima facies semble vouloir réintroduire des principes altruistes de mutualisation des ressources face à la privatisation du vivant, repose en fait sur une conception libertarienne de la justice qui laisse aux opérateurs industriels une totale liberté en matière d'accès aux ressources génétiques sans aucune contrepartie de partage. Ainsi, alors que le protocole de Nagoya travaille pour que les contrats de partage des avantages jouent tant soit peu un rôle de compensation au développement de la propriété intellectuelle sur le vivant (Thomas, 2012), la FAO s'interdit de jouer un tel rôle tant quelle érige les ressources génétiques agricoles en un bien public mondial qui empêche la reconnaissance des droits collectifs des communautés rurales sur leurs variétés locales. Il y a ici une question de philosophie morale et politique de la gouvernance du vivant pour articuler le domaine public, les biens collectifs et les biens privés, et qui revient à se demander quel est l'outil le plus efficace de rééquilibrage des excès des tendances à l'exclusivisme de la propriété industrielle : renforcer les mécanismes contractuels de compensation (Biber-Klemm et Cottier, 2005) : xxiv; Bellivier et Noiville, 2006) ou reconstituer des commons pour limiter létendue de la propriété intellectuelle (Cassier, 2002)?

\section{CONCLUSION}

Deux conceptions de la justice sont donc à l'œuvre dans les deux régimes d'accès à la biodiversité et de partage des avantages qui en découlent que nous venons d'examiner. Le régime "général» institué par la CBD est clairement basé sur une conception rawlsienne de la justice qui fait du marché le meilleur instrument de distribution des avantages tirés de la mise en valeur de la biodiversité à condition que les droits de chacun soient assurés par des institutions, ici précisément le régime d'accès et de 
partage de la $\mathrm{CDB}$ et du protocole de Nagoya. Ce régime, en dépit de la faiblesse des sommes redistribuées aux populations par rapport aux bénéfices engendrés, est bel et bien redistributif.

Dans le régime institué par le Tirpaa, le système multilatéral de la FAO maintient les ressources génétiques agricoles dans un «commun techno-scientifique mondialisé» dont l'accès est gratuit pour les États membres, donc sans contrepartie pour les paysans (nonobstant le symbolique fonds de partage des avantages). La FAO fait valoir pour s'en justifier que le brassage du génome des plantes cultivées à l'échelle de la planète rend impossible la possibilité d'attribuer à des agriculteurs de quelconques droits de propriété sur les variétés qu'ils cultivent. Il paraît impossible dans ces conditions d'imaginer un statut, même collectif, d'agriculteurspourvoyeurs, nécessaire à la mise en place de contrats de partage des avantages. Le Tirpaa fait donc des ressources génétiques agricoles un bien public mondial tout en reconnaissant la souveraineté des États sur ces ressources, ce qui permet de les maintenir sur le plan national dans la règle des choses sans maître (resnullius) appropriable par le premier disant. Les variétés paysannes et les races animales locales, autrefois «communs» sur lesquels les communautés locales avaient établi des règles d'usage, sont ainsi redéfinies en droit de la propriété intellectuelle comme relevant du domaine public, ce qui permet de nier tout droit collectif sur ces variétés aux communautés paysannes et d'en faire ainsi des ressources génétiques en libre accès pour que les généticiens-sélectionneurs en tirent des innovations végétales appropriables soit par brevet, soit par COV. Plus que le développement de la propriété privée, c'est donc la constitution d'un domaine public, réservoir des futures innovations industrielles appropriables, qui empêche la reconnaissance des droits des communautés rurales sur les variétés locales. Le principe de la mutualisation des ressources génétiques agricoles empêche donc fondamentalement la mise en place d'un régime de partage des avantages par contrat et c'est la liberté d’opérer des scientifiques et des industriels qui est ainsi assurée.

Pour sortir de cette conception libertarienne de la justice, la piste la plus sérieuse (et qui est mentionnée dans le préambule du Tirpaa) serait de permettre aux agriculteurs de produire, d'échanger et de multiplier leurs semences de ferme. Ce serait le moyen le moins cher et le plus efficace pour mettre en place un partage global des avantages de l'amélioration de 
la biodiversité cultivée. Cette manière de penser le partage des avantages pour les ressources génétiques agricoles supposerait de sortir du cadre strict de la propriété industrielle pensant uniquement la rémunération de l'effort de recherche, pour le replacer dans le système global de l'amélioration des plantes. Le partage des avantages deviendrait alors un élément de la répartition de la rente du progrès génétique, dont on reconnaîtrait qu'elle ne peut pas aller exclusivement vers les détenteurs des titres de propriété, car ils ne sont qu'un chaînon du processus constant d'évolution et d'amélioration des plantes cultivées dans des environnements économiques et culturels mouvants. Dans ce cadrage plus systémique, plus évolutionniste des ressources génétiques (Bonneuil et Fenzi, 2011), il ne serait alors plus légitime de considérer les semences de ferme comme une violation des droits de propriété industrielle des sélectionneurs. Cette concurrence deviendrait tout simplement le prix du partage des avantages le prix d'une vision plus équitable de la répartition de la rente. La FAO pourrait alors justifier cette conception du partage des avantages en montrant qu'il s'agit d'un «agencement distributif » (Hayden, 2007: 748) sui generis aux ressources génétiques agricoles et à l'amélioration des plantes, tout en le fondant sur la théorie de la justice de Rawls en affirmant que la liberté des agriculteurs de produire leurs semences est juste, car elle rétablit une égalité des droits entre les agriculteurs et les industriels: le libre accès aux ressources génétiques contenues dans les variétés paysannes pour les industriels ne pouvant se justifier que pour un principe d'équivalence de libre accès des agriculteurs aux ressources génétiques contenues dans les variétés industrielles.

Il reste enfin à nous interroger sur l'efficacité de ces deux conceptions du partage des avantages, non pas seulement sous l'angle de justice, mais aussi sous l'angle de léthique de l'environnement en nous demandant en quoi la conception du libre accès du Tirpaa produit des effets bénéfiques en matière de conservation de la biodiversité. Si l'on voit bien comment la conception rawlsienne du partage des avantages de la $\mathrm{CDB}$ peut théoriquement permettre des retours vers des populations rurales ou forestières pauvres, qui produisent des effets bénéfiques sur la conservation des éléments de la biodiversité locale, et ce, au nom de la valeur intrinsèque de ses éléments ou des écosystèmes affirmée dans le préambule de la $\mathrm{CDB}$, on voit mal, en revanche, comment la conception libertarienne du libre accès 
aux ressources génétiques agricoles peut produire de tels effets. En fait, le Tirpaa n'a permis que de repréciser les règles d'accès à un réseau de banques de gènes ex situ pour faciliter l'accès des sélectionneurs du monde entier au matériel génétique des plantes cultivées, mais il n'est pas parvenu à y inclure les paysans. De fait, il est quasiment sans effet sur la conservation in situ de la biodiversité cultivée (sauf les quelques programmes financés par le fonds de partage). Malheureusement, le Tirpaa a finalement conduit à soustraire les ressources génétiques pour l'agriculture et l'alimentation des modestes avancées de la $\mathrm{CDB}$ en matière de justice et d'éthique environnementale.

\section{BIBLIOGRAPHIE}

Armstrong, Adrian (2014), Ethics and Justice for the Environment, Routledge.

Andersen, Regine (2008), Governing Agrobiodiversity: Plant Genetics and Developing Countries, Ashgate, Aldershot, Serie Global Environmental Governance.

Bellivier, Florence, et Christine NoIVILle (2006), Contrats et vivant. Le droit de la circulation des ressources biologiques, Paris, L.G.D.J.

BENTON, Ted (1997), «Ecology, community and justice», dans T. HAYWARD et J. O'NeILl (dir.), Justice, Property and the Environment: Social and Legal Perspectives, Ashgate, Sydney, Aldershot.

BENTON, Ted (1999), «Sustainable development and accumulation of capital: reconciling the irreconcilable?", dans Andrew DobSON (dir.), Fairness and Futurity: Essays on Environmental Sustainability and Social Justice, Oxford, Oxford University Press, p. 199-229.

Biber-Klemm, Susette, et Thomas CotTier (dir.) (2006), Rights to Plants Genetic Resources and Traditional Knowledge Basic Issues and Perspectives, CABI.

Blanchon, David, et al. (2009), «Comprendre et construire la justice environnementale », Annales de géographie, n ${ }^{\text {os }}$ 665-666, p. 35-60.

Bonneuil, Christophe, et Marianna FenZI (2011), «Des ressources génétiques à la biodiversité cultivée. La carrière d'un problème public mondial », Revue d'anthropologie des connaissances, vol. 5, n 2, p. 206-233. 
Bonneull, Christophe, et Frédéric ThOMAs (2009), Gènes, pouvoirs et profits. Recherche publique et transformations des régimes de production des savoirs et des innovations en génétique végétale de Mendel aux OGM, Éditions Quae et Fph.

Buck, Matthias, et Clare Hamilton (2011), «The Nagoya Protocol on access to genetic resources and the fair and equitable sharing of benefits arising from their utilization to the Convention on Biological Diversity", Review of European Community $\Xi^{\circ}$ International Environmental Law, vol. 20, $\mathrm{n}^{\circ} 1$, p. 47-61.

Callicott, Baird J. (2010), Éthique de la Terre. Philosophie de l'écologie, Wildproject.

CASsiER, Maurice (2002), «Bien privé, bien collectif et bien public à l'âge de la génomique ", Revue internationale des sciences sociales, no 171, p. 95-110.

Dobson, Andrew (1998), Justice and the Environment: Conception of Environmental Sustainability and Theories of Distributive Justice, Oxford, Oxford University Press.

Dobson Andrew (1999), Fairness and Futurity. Essays on Environmental Sustainability and Social Justice, Oxford, Oxford University Press.

Fowler Cary, et Toby Hodgkin (2004), « Plant genetic resources for food and agriculture : Assessing global availibility», Annual Review of Environment and Resources, vol. 29, p. 143-179.

GirarD, Florent, et Christine NoIVILLE (dir.) (2013), Biotechnologies végétales et propriété industrielle, rapport du groupe de travail mis en place par le Comité économique, éthique et social (CEES) Haut Conseil des Biotechnologies, Paris, 4 avril 2013, [En ligne], [http://www.hautconseildesbiotechno logies.fr/IMG/pdf/130612_Propriete_industrielle_Rapport_Groupe_de_tr avail_HCB.pdf], (23 mars 2014).

Hache, Émilie (2011), Ce à quoi nous tenons. Propositions pour une écologie pragmatique, Paris, La Découverte. (Coll. "Les empêcheurs de penser en rond ».)

Hayden, Cori (2003), When Nature Goes Public. The Making and Unmaking of Bioprospecting in Mexico, Princeton, Princeton University Press.

HaYden, Cori (2007), «Taking as giving: Bioscience, exchange, and the politics of benefit-sharing », Social Studies of Science, vol. 37, $\mathrm{n}^{\circ}$ 5, p. 729-758.

Hess, Gérald (2013), Éthiques de la nature, Paris, Presses universitaires de France. 
Hermitte, Marie-Angèle, et Philippe Kahn (dir.) (2004), Les ressources génétiques végétales et le droit dans les rapports Nord-Sud, Bruxelles, Bruylant.

Jonas, Hans (1979), Le principe de responsabilité. Une éthique pour la civilisation technologique, Paris, Flammarion.

LANGHELle, Oluf (2000), "Sustainable development and social justice: expanding the Rawlsian framework of global justice», Environmental Values, vol. 9, n 3, p. 295-323.

LARRÈRE, Catherine (2009), «La justice environnementale », Multitudes, no 36, p. 156-162.

LARRÈRE, Catherine (2010), «Les éthiques environnementales », Natures Sciences Sociétés, vol. 18, no 4, p. 405-413.

LARRÈRE, Catherine, et Raphaël LARRÈRE (1997), Du bon usage de la nature. Pour une philosophie de l'environnement, Paris, Aubier.

NIJAR, Gurdial Singh (2011), «Food security and access and benefit sharing laws relating to genetic resources: promoting synergies in national and international governance», International Environmental Agreements, vol. 11, $\mathrm{n}^{\circ} 2$, p. 99-116.

Nozick, Robert (1974), Anarchy, State and Utopis, New York, Basic Books.

OKEREKe, Chukwumerije (2006), «Global environmental sustainability: intragenerational equity and conceptions of justice in multilateral environmental regimes », Geoforum, vol. 37, n 5, p. 725-738.

PucketT, Jim (1992), «Dumping on our world neighbours», dans H.O. Bergesen, M. Norderhaug et G. Parmann (dir.), Green Globe Yearbook, Oxford, Oxford University Press, p. 93-106.

RAwLS, John (1971), A Theory of Justice, Oxford, Oxford University Press.

SAndel, Micheal J. (1982), Liberalism and the Limits of Justice, Cambridge, Cambridge University Press.

SHIVA, Vandana (1997), Biopiracy. The Plunder of Nature and Knowledge, South End Press.

Thomas, Frédéric (2006), «Biodiversité, biotechnologies et savoirs traditionnels. $\mathrm{Du}$ patrimoine commun de l'humanité aux ABS», Revue Tiers Monde, vol. $47, \mathrm{n}^{\circ} 188$, p. $825-842$.

Thomas, Frédéric (2012), «Le protocole de Nagoya au secours des contrats d'accès à la biodiversité », Revue des contrats, juillet, p. 961-972. 
ThOMAs, Frédéric, et Valérie BOISVERT (à paraître), Le pouvoir de la biodiversité, Éditions de l'IRD.

VIOTTI, Paul R., et Mark V. KAUPPI (1987), International Relations Theory: Realism, Pluralism and Globalism, New York, Macmillan.

VIVAs-Eugu, David (2012), Bridging the Gap on Intellectual Property and Genetic Resources in WIPO's Intergovernmental Committee (IGC), ICTSD's Programme on Innovation, Technology and Intellectual Property, Issue no 34 ; International Centre for Trade and Sustainable Development, Genève.

Young, Iris Marion (1990), Justice and the Politics of Difference, Princeton, Princeton University Press. 